\title{
LIDERAZGO Y CONOCIMIENTO COMPARTIDO EN CONTEXTOS INTERORGANIZACIONALES*
}

\author{
GUSTAVO ADOLFO ESGUERRA PÉREZ" \\ UNIVERSIDAD DEL ROSARIO
}

Recibido/ Received/ Recebido: 02/03/2016-Aceptado/ Accepted / Aprovado: 24/06/2016

\begin{abstract}
Resumen
Este documento presenta una descripción teórica, desde la perspectiva relacional, de uno de los factores determinantes de ventaja competitiva: las rutinas de conocimiento compartido (Dyer \& Singh, 1998), las cuales se consideran fundamental en el logro de rentas relacionales, y se revisan algunos elementos dirigidos a comprender su desarrollo. También se describe la función del liderazgo entendido como el ejercicio deliberado de influencia para el logro de objetivos (Huxham \& Vangen, 2000), en este caso, compartir conocimiento. Se proponen además, los tipos y características de liderazgo que pueden emerger específicamente en el proceso de transferencia y compartimiento de conocimiento interorganizacional, desde las propuestas de Cox, Pearce \& Sims (2011) y Uhl-Bien \& Marion (2008). Para terminar se señalan las implicaciones y perspectivas que de este trabajo se desprenden para la investigación en el campo.

Palabras clave: Liderazgo; Conocimiento compartido; Redes; Relaciones interorganizacionales; Cooperación.

\section{LEADERSHIP AND KNOWLEDGE SHARED IN INTER-ORGANIZATIONAL ENVIRONMENTS}

\begin{abstract}
This paper presents a theoretical description, from the relational perspective of one of the competitive advantage decisive elements: shared knowledge routines (Dyer \& Singh, 1998), which are considered critical in achieving relational rents, and some elements are reviewed; aimed at understanding their development. The role of leadership understood as the deliberate exercise of influence to achieve goals (Huxham \& Vangen, 2000), in this case, sharing knowledge is also described. The types and characteristics of leadership that can emerge specifically in the transfer process and sharing of inter-organizational knowledge are also proposed. From the recommendations of Cox, Pearce \& Sims (2011) and Uhl-Bien \& Marion (2008). Finally the implications and perspectives of this work are indicated for research in the field.

Keywords: Leadership; Shared knowledge; Networks; Inter-organizational relationships; Cooperation.
\end{abstract}

Artículo de refglexión derivado del proyecto de investigación: "Comportamiento organizacional, liderazgo, bienestar; cultura, emprendimiento, y calidad de vida laboral". Entidad: Universidad del Rosario - Escuela de Administración.

Profesor Principal de la Escuela de Administración de la Universidad del Rosario. Dirección postal: Autopista Norte - Calle 200 (Módulo A) (Bogotá). Teléfono: (57 -1) 2970200 Ext. 3957. Correo electrónico: esguerra.gustavo@urosario.edu.co 


\title{
LIDERANÇA E CONHECIMENTO COMPARTILHADO EM CONTEXTOS INTERORGANIZACIONALES
}

\begin{abstract}
Resumo
Este documento apresenta uma descrição teórica, desde a perspectiva relacional, de um dos fatores determinantes de vantagem competitiva: as rotinas de conhecimento compartilhado (Dyer \& Singh, 1998), as quais se consideram fundamentais no alcance de rendas relacionais, e se revisam alguns elementos dirigidos a compreender seu desenvolvimento. Também se descreve a função da liderança entendida como o exercício deliberado de influência para o alcance de objetivos (Huxham \& Vangen, 2000), neste caso, compartir conhecimento. Propõem-se além do mais, os tipos e características de liderança que podem emergir especificamente do processo de transferência e compartilhamento de conhecimento interorganizacional, desde as propostas de Cox, Pearce \& Sims (2011) e Uhl-Bem \& Marion (2008). Para terminar indicam-se os envolvimentos e perspectivas que deste trabalho se desprendem para a pesquisa no campo.

Palavras chave: Liderança; Conhecimento compartilhado; Redes; Relaciones interorganizacionais; Cooperação.
\end{abstract}

Esguerra, G. (2017). Liderazgo y conocimiento compartido en contextos interorganizacionales. En: Revista de la Facultad de Ciencias Económica: Investigación y Reflexión. rev.fac. cienc.econ, XXV (1). DOI: http://dx.doi.org/10.18359/rfce.1768

JEL: M19, M54.

\section{Introducción}

La estrategia de establecimiento de relaciones colaborativas, de cooperación y de redes interorganizacionales en diferentes sectores ha venido incrementándose paulatinamente y consolidándose como una forma de organización de la producción que genera efectos favorables en el desempeño de las empresas.

Varios autores se han referido a las probables ventajas competitivas derivadas de estas interrelaciones tales como; optimización en el acceso a recursos, orientación estratégica, reducción de costos, aumento de la eficiencia, satisfacción de clientes, compartimiento de costos y riesgos, acceso a nuevos mercados y aceleración de ciclos de innovación de productos (Barringer \& Harrison, 2000; Doz \& Hamel, 1998; Grueso, Gómez \& Garay, 2010; Lavie, 2006; Muller, 2012, Powell, Koput, \& SmithDoerr, 1996; Sánchez \& Jiménez, 2007; Scott \& Thomas, 2015).
Para la investigación sobre este tipo de estrategia, la propuesta de Dyer \& Singh (1998), ha provisto de un marco metodológico para analizar los elementos de la ventaja competitiva, señalando como factores determinantes los activos de la relación, los recursos-capacidades complementarias, la gobernanza efectiva y las rutinas de conocimiento compartido.

Con respecto al último factor, se han reportado como asociadas a este, la generación de rentas relacionales tales como; el intercambio de información, el fortalecimiento de la investigación, el desarrollo del saber cómo (know -how), el aprendizaje organizacional y la transferencia de conocimiento (Dyer \& Singh, 1998; Lavie, 2006; Levinson \& Asahi, 1996; Mesquita, Anand \& Brush 2008; Müller, 2012; Powell, Koput \& Smith-Doerr, 1996).

A partir de estos elementos, el objetivo de este artículo se dirige a presentar de manera general la perspectiva relacional desde la propuesta de Dyer \& 
Singh (1998), haciendo énfasis en el factor determinante de rentas relacionales, específicamente, las rutinas de conocimiento compartido. A continuación, se abordará el tema del conocimiento y la transferencia del mismo en las redes interorganizacionales.

Posteriormente, se señalará la importancia de los procesos de dirección y liderazgo en la generación de conocimiento compartido y se presentarán los posibles tipos y características de liderazgo que pueden emerger entorno a la producción de conocimiento en contextos interorganizacionales. Finalmente se sugerirán las implicaciones académicas y perspectivas de investigación.

\section{La perspectiva relacional}

Dentro del análisis de la estrategia en el campo de la dirección, la perspectiva relacional es una propuesta teórica en la cual se consideran las redes de empresas como unidades de análisis (Barringer \& Harrison, 2000; Verdu \& Reinert, 2016) y sobre las que se concentra la explicación de la obtención de ventaja competitiva y de rentas relacionales (Matinheikki, Artto, Peltokorpi \& Rajala, 2016).

De acuerdo con Dyer \& Singh (1998); las rentas relacionales se entienden como el beneficio extraordinario generado conjuntamente en una relación de intercambio, que no puede producirse por alguna de las firmas aisladamente sino que requiere la suma de las contribuciones particulares y especializadas de los miembros de la relación. Es decir un desempeño superior de la empresa generado por la participación en una red.

Según Lavie (2006), la perspectiva relacional complementa las perspectivas existentes. Mientras la perspectiva de la estructura de la industria (Porter, 1980), explica los rendimientos superiores como producto de la participación de una empresa en una industria con características específicas estructurales y la perspectiva basada en recursos (Barney, 1991; Rumelt, 1991), los explica en función de heterogeneidad, los recursos y las capacidades de la firma.

Desde el punto de vista relacional, la renta relacional solo puede ser creada a través de las contribuciones conjuntas idiosincrásicas de los socios de una alian- za. Así mismo, las ventajas y rentas relacionales están determinadas fundamentalmente por cuatro factores:

- Los activos de la relación con sus subprocesos de establecimiento de salvaguardas y de volumen de transacciones interfirma.

- Los recursos-capacidades complementarios, con sus subprocesos de habilidad para identificar y evaluar las posibles complementariedades y el rol de las complementariedades para acceder a los beneficios estratégicos.

- La gobernanza efectiva con sus subprocesos de habilidad para emplear el cumplimiento y el compromiso autoimpuesto, más que la imposición del cumplimiento por parte de terceros y la habilidad para emplear mecanismos informales de gobierno que emergen de la relación.

- Las rutinas de conocimiento compartido con sus subprocesos de capacidad absorbente para aprender de los aliados y el establecimiento de incentivos para fomentar la transparencia y disuadir del aprovechamiento o beneficios sin compromiso (free riding) (Dyer \& Singh, 1998).

\section{El conocimiento en las redes interor- ganizacionales}

Dyer \& Nobeoka (2000) y Dyer \& Singh (1998), citan y se refieren a las rutinas de conocimiento compartido partiendo de lo que académicos como March \& Simon (1958), Levinson \& Asahi, (1996) y Powell, Koput \& Smith-Doerr (1996), habían propuesto previamente, al afirmar que el aprendizaje interorganizacional es fundamental para el éxito de la ventaja competitiva.

Por lo tanto, se señala que las organizaciones fortalecen el aprendizaje por colaborar con otras organizaciones, que la innovación está asociada a las rutinas de intercambio de conocimiento, que muchas de las patentes son producto de trabajo de individuos de diferentes organizaciones trabajando en conjunto y que las firmas incapaces de crear redes de aprendizaje o conocimiento compartido tienen una desventaja competitiva en el mercado. 
Además se propone que a mayor inversión de rutinas de conocimiento en la alianza, mayor es el potencial de obtener rentas relacionales; que a mayor capacidad absorbente o de aprendizaje de una empresa participante, mayor es el potencial de generar rentas relacionales a través del compartir conocimiento y que a mayor ajuste de incentivos por aliarse para fomentar transparencia y reciprocidad así como para disuadir el "ir en coche" (free riding), mayor es el potencial de generar rentas relacionales a través del compartir conocimientos (Dyer \& Singh, 1998; Dyer \& Nobeoka, 2000).

Las rutinas se consideran entonces como patrones de interacción entre las empresas y se diseñan para facilitar la transferencia de conocimiento especializado, tanto explícito como tácito, entre los miembros de la relación.

El conocimiento explícito se caracteriza por ser formal y sistemático, fácil de comunicar y compartir, mientras que el conocimiento tácito es altamente personal, muy difícil de articular y expresar y consistente en modelos mentales, creencias y perspectivas que dirigen la acción del experto (Dyer \& Nobeoka, 2000; Dyer \& Singh, 1998).

Así miso, las rutinas se caracterizan por ser interacciones directas entre los individuos, las cuales requieren contacto personal para intercambiar el conocimiento (Dyer \& Nobeoka, 2000). Por ejemplo, en las rutinas que se utilizan para transmitir conocimiento explícito, la interacción y el contacto personal son de baja frecuencia e intensidad, lo que puede incluir reuniones en los rangos directivo y operativo, visitas a plantas, cursos de capacitación y conferencias principalmente.

Por otra parte, puede considerarse a las rutinas como el único modo de transferencia de conocimiento tácito, llevado a cabo mediante interacciones y contacto personal frecuente $e$ intenso, en forma por ejemplo de cursos de entrenamiento, talleres prácticos, asistencia técnica, equipos de mejoramiento e incluso, de transferencia de empleados (Dyer \& Nobeoka, 2000; Inkpen \& Dinur, 1998).

Algunos estudios que han explorado el papel de las actividades relacionadas con el conocimiento, se han centrado en describir cómo es posible el intercambio de conocimientos entre los socios de la red.

El estudio de Dyer \& Nobeoka (2000), señaló la importancia de establecer rutinas de intercambio de conocimientos asociadas al desarrollo de la confianza entre las organizaciones participantes, así como de factores relevantes para la reducción de costos y la prevención del "ir en coche" (free riding).

En una línea similar, Dhanaraj \& Parkhe (2006), argumentaron que la orquestación y estabilidad de redes de conocimiento favorece, al permitir la difusión del conocimiento, la optimización de la innovación; ideas que son corroboradas por la evidencia hallada en redes entre corporaciones multinacionales, lo que da cuenta de la relación entre conocimiento compartido y la innovación asociada al mejoramiento en rankings empresariales, conducente al liderazgo en los respectivos sectores (Inkpen \& Dinur, 1998; Müller, 2012; Mursitama, 2006).

Por su parte, Häcki \& Lighton (2001) y Nambisan \& Sawhney (2007), argumentaron que los orquestadores o líderes de la interrelación deben diseñar redes que permitan mantener y fortalecer oportunidades para el intercambio de conocimientos mediante procedimientos como la instalación de plataformas electrónicas y el aprovechamiento de las tecnologías de comunicación, hallazgo que también se ha confirmado para las pequeñas y medianas redes empresariales (Dini, 2010).

Una vez reconocidas las condiciones y factores bajo las que se da el conocimiento compartido la pregunta por la dirección de las actividades y las rutinas, conduce a señalar el papel del liderazgo en estos procesos.

Es evidente que las redes interorganizacionales no cumplen con las mismas condiciones de las organizaciones individuales, por lo tanto, liderazgo ha de ser analizado bajo la condición y el contexto interorganizacional en el que una red de organizaciones independientes se establece temporal o permanente y es percibida y aceptada por los demás organizaciones participantes (Huxham \& Vangen 2000; Scott \& Thomas, 2015).

Martín et al., (2008) sugirieron al respecto, que con el fin de dirigir la red, los respectivos líderes deben 
ocupar puestos de responsabilidad dentro de su organizaciones de procedencia o de base, así como en la red a la que están asignados.

\section{Liderazgo en la generación de conoci- miento compartido}

Para la descripción y comprensión del fenómeno del liderazgo, entendido como el ejercicio deliberado de influencia para el logro de objetivos (Yukl, 2006) y "hacer que las cosas pasen" (Huxham \& Vangen, 2000), se han presentado a lo largo de los varios años de investigación diferentes teorías entre las que se destacan la del "gran hombre", la de los rasgos, las conductuales o de estilos, las contextuales y las de contingencia o emergentes (Arbaiza, 2009; Contreras, 2008; Dinh et al., 2014; Huxham \& Vangen 2000).
Desde tales perspectivas, por lo general los procesos organizacionales son analizados en función de lo que son o hacen los directivos al interior de la organización en particular, observándose de manera aislada y en las que las relaciones entre organizaciones a lo sumo se perciben dentro de una concepción de gremios más que como red de organizaciones.

Por otra parte, en cuanto al estudio de la relación entre liderazgo y la generación de conocimiento compartido en contextos interorganizacionales, los reportes de investigación son más bien escasos y principalmente de carácter teórico (Müller, 2012).

Sin embargo, algunas aproximaciones han dado cuenta principalmente de las habilidades que deben tener los líderes entre las que se encuentran las que se presentan en la tabla 1.

Tabla 1. Habilidades que deben tener los líderes

\begin{tabular}{|c|c|}
\hline Autor & Habilidades del líder \\
\hline Galaskiewicz \& Shatin (1981) & $\begin{array}{l}\text { Reconocieron que en las organizaciones se adquiere el conocimiento tácito con el tiempo, el cual no } \\
\text { puede ser adoptado rápidamente como el conocimiento explícito e identificaron los lazos interper- } \\
\text { sonales que facilitan el inicio de la interacción entre organizaciones las relaciones a largo plazo y la } \\
\text { responsabilidad de los líderes en estos aspectos. }\end{array}$ \\
\hline $\begin{array}{l}\text { Tushman \& Scanlan (1981, citados por } \\
\text { Doshi \& Khokle, 2011) }\end{array}$ & $\begin{array}{l}\text { Describieron la función de los líderes en la obtención de conocimiento desde fuera de la organización y } \\
\text { en la difusión de los conocimientos a los usuarios internos. }\end{array}$ \\
\hline $\begin{array}{l}\text { Nonaka \& Takeuchi (1995, citados por Do- } \\
\text { shi \& Khokle, 2011) }\end{array}$ & $\begin{array}{l}\text { Indican el rol de los directivos en la formación de las relaciones inter-organizacionales para el aprendi- } \\
\text { zaje, en la promoción de interacciones para incrementar los conocimientos existentes de las organiza- } \\
\text { ciones, así como, las reservas de conocimientos, sinergias y economías de escala. }\end{array}$ \\
\hline $\begin{array}{l}\text { Watkins \& Marsick (1996, citados por Do- } \\
\text { shi \& Khokle, 2011) }\end{array}$ & $\begin{array}{l}\text { Identificaron varias dimensiones de la organización aprendizaje, tales como el aprendizaje continuo, } \\
\text { la investigación y el diálogo, el aprendizaje en equipo, el empoderamiento, el sistema integrado, la } \\
\text { conexión del sistema y propusieron el liderazgo estratégico como estilo favorable en las relaciones } \\
\text { interorganizacionales de conocimiento. }\end{array}$ \\
\hline Kraatz (1998) & Describió las ventajas de liderar procesos de aprendizaje para adaptarse al cambio. \\
\hline Barringer \& Harrison (2000) & $\begin{array}{l}\text { Dieron cuenta de la adaptación que deben tener los líderes al proveer y recibir conocimientos en condi- } \\
\text { ciones de interrelación y sus posibles riesgos organizacionales. }\end{array}$ \\
\hline Dyer \& Nobeoka (2000) & $\begin{array}{l}\text { Reportaron la relación positiva entre las prácticas de liderazgo y el uso y apropiación del saber compar- } \\
\text { tido en redes interorganizacionales, a partir de un caso en la empresa Toyota. }\end{array}$ \\
\hline Knight (2002) & $\begin{array}{l}\text { Describió el rol de los líderes en la generación de aprendizaje organizacional y hace una diferenciación } \\
\text { entre momentos de aprendizaje de acuerdo al desarrollo de la relación. }\end{array}$ \\
\hline Vicente \& Byrne (2006) & $\begin{array}{l}\text { Revisaron los factores de flexibilidad, apreciación de la diversidad, apertura, confianza y respeto mutuo } \\
\text { como fundamentales en desarrollo de relaciones interorganizacionales. }\end{array}$ \\
\hline Peroune (2007) & Reportó el conocimiento tácito en función del tiempo, la adaptación y la sinergia de las relaciones. \\
\hline
\end{tabular}

Fuente: Elaboración propia. 
Como se identifica en esta revisión (tabla 1) sobre el estudio del liderazgo en la generación de conocimiento compartido se puede observar una incipiente conceptualización, lo que posiblemente no facilita la comprensión de las variables que determinan este fenómeno.

Si bien se han desarrollado perspectivas y modelos para comprender las interacciones y las redes interorganizacionales en sus diversas formas, es claro que se requiere de mayor estructuración metodológica, así como de propuestas que favorezcan la descripción, la identificación de relaciones y la proposición de estudios empíricos entorno a la dinámica del liderazgo en estos contextos así como de los efectos y relaciones del liderazgo con cada uno de los factores determinantes de ventaja competitiva y rentas relacionales, entre los que el aprendizaje y el conocimiento compartido son fundamentales.

\section{Tipos y características de liderazgo en la generación de conocimiento com- partido}

Se propone para el análisis de la generación de conocimiento compartido en contextos interorganizacionales, una aproximación basada en la perspectiva de Cox, Pearce \& Sims (2011) quienes parten de la revisión de varios modelos, estudios y propuestas sobre liderazgo que se han presentado en los últimos años de análisis en el campo, agregando un estilo más, el de liderazgo complejo de Uhl-Bien \& Marion (2008).

La tipología de Cox, Pearce \& Sims (2011) incluye cuatro tipos de liderazgo; directivo, transaccional, transformacional y de empoderamiento y se sintetiza a partir de las diferentes teorías que en el pasado se han formulado en la investigación.

A la propuesta se suma un tipo más; el liderazgo complejo, por considerar la interrelaciones como sistemas adaptativos complejos en los que la emergencia de condiciones y situaciones impredecibles son altamente probables (Arbaiza, 2009; Uhl-Bien \& Marion, 2008).
Se considera útil esta clasificación por presentar los comportamientos que están asociados a cada tipo de liderazgo y puede ser favorable para identificar un tipo particular de conductas de liderazgo de acuerdo con los diferentes momentos o circunstancias en que la relación interorganizacional, y por consiguiente el proceso de generaciones de conocimiento se encuentra.

El primero, el liderazgo directivo, describe el liderazgo que inicialmente depende de la posición de poder o del poder legítimo y puede incluir también el poder coercitivo. Esta aproximación asume que el comportamiento del seguidor es a través de los comportamientos del líder tales como mandar, asignar objetivos, capacitar, conducir.

La raíz del liderazgo directivo aquí, se encontraría en la llamada Teoría X, en la Teoría de Iniciación a la Estructura de los estudios de Ohio State y en la de Orientación a la Tarea identificada por los estudios de la Universidad de Michigan, así como en los análisis sobre la coerción (McGregor, 1960; House \& Kerr, 1976; Likert, 1967; Sims, 1980, Quinn, 1990; Kazdin 1975). Los comportamientos que representan este tipo de liderazgo son principalmente la emisión de mandos, la asignación de metas y el uso del poder.

Las situaciones en la que se demandaría de este estilo de liderazgo serían en la fase temprana de conformación de grupos o redes, en las situaciones de dirección y establecimiento de acuerdos de colaboración, cuando hay procesos y participantes nuevos en una tarea u objetivo, cuando los consensos son difíciles de establecerse y cuando los hay cierto grado de subordinación.

En cuanto a las rutinas de conocimiento compartido a las que se aplicaría este tipo de liderazgo sería a las de interacción directa entre los individuos, de contacto personal para intercambiar el conocimiento (Dyer \& Nobeoka, 2000), así como a las rutinas que se utilizan para transmitir conocimiento explícito en las cuales se requiere alta intensidad, coerción y convencimiento de los participantes, lo que incluiría dentro de las rutinas; las reuniones de instruccionales, las reuniones de dirección y opera- 
ción, las visitas a plantas, los cursos de capacitación y conferencias.

El segundo tipo es el transaccional. Este tipo de liderazgo incluye comportamientos de hacer acuerdos y transacciones, monitoreo de las transacciones y provisión de recompensas contingentemente. La base de este tipo de liderazgo está principalmente en la Teoría de la Expectativa, la Teoría del Camino Meta y los principios de intercambio/equidad (Bass, 2005; House, 1971; Vroom, 1964; Homans; 1961; Sims, 1977).

Las situaciones en las que se demanda de este tipo de liderazgo son principalmente cuando se tienen una expectativa de recompensa, cuando se necesita evaluar el desempeño y cuando es necesario dar recompensas o reconocimientos por el desempeño.

Así mismo, cuando para alcanzar las metas organizacionales y guiar el desempeño de los participantes se deben hacer acuerdos y establecer funciones con asignación de tareas específicas. Aquí también, se presentan más conductas de monitoreo y control de las actividades de los colaboradores procurando evitar posibles errores en los procedimientos establecidos.

En cuanto al establecimiento de conocimiento compartido el liderazgo transaccional, se requerirá en las fases de la relación cuando las rutinas son el único modo de transferencia de conocimiento principalmente el de carácter tácito.

De acuerdo con Dyer \& Nobeoka (2000) y Inkpen \& Dinur (1998) las acciones de liderazgo implicarían interacciones y contacto personal, negociaciones y también incluiría en la trasferencia de saber, la inclusión de cursos de entrenamiento, talleres prácticos, seguimiento cercano y evaluación, asistencia técni$\mathrm{ca}$, equipos de mejoramiento y la transferencia de empleados entre las organizaciones participantes.

El tercer tipo es el liderazgo transformacional, que tiene como principales comportamientos el proveer visión, expresar ideales, comunicación y generar inspiración. Las situaciones en que se valora en contextos interorganizacionales es cuando se ne- cesita unificar un grupo alrededor de un propósito (Bass \& Riggio, 2006).

El liderazgo transformacional, está en función de los efectos y cambios que genera el líder sobre el comportamiento de las personas e identifica que los líderes que muestran características transformacionales producen los cambios en los seguidores con base en la toma de conciencia sobre los valores y la importancia que tienen los resultados de la tarea que se realiza o de las responsabilidades encargadas (Bass \& Riggio, 2006; Castro, 2006; Mendoza, Ortiz \& Parker, 2007).

En este tipo se asume que el líder conduce a los colaboradores se involucren con sus intereses personales en los objetivos del grupo y de la organización, lo que se ha relacionado con la confianza, la autoeficacia, la motivación al trabajo y el rendimiento laboral y que se ha evidenciado es mas más efectivo que el de tipo transaccional, en el que fundamentalmente se establecen compromisos de tipo negociación de responsabilidades y resultados (Lupano \& Castro, 2005; García, Jiménez \& Gutiérrez, 2012; Paglis \& Green, 2002).

Este liderazgo en el proceso de conocimiento compartido, debería surgir adaptativamente en situaciones de fortalecimiento de relaciones de largo plazo, cuando se necesita cambiar el curso de una acción y cuando se reta el status en la interrelación.

De acuerdo con el estudio de Dyer \& Nobeoka (2000) este tipo de liderazgo fundamentaría la importancia de establecer rutinas de intercambio de conocimientos asociadas al desarrollo de la confianza entre las organizaciones participantes de una red así como de factores relevantes para la reducción de costos y la prevención de free riding.

Así mismo favorece la orquestación y estabilidad de redes de conocimiento, de acuerdo con Dhanaraj \& Parkhe (2006), al permitir la difusión del conocimiento, la optimización de la innovación, el rendimiento, el fortalecimiento de valores y el incremento de la importancia que tienen los resultados de las tareas que se realizan, así como de las responsabilidades encargadas, de los objetivos del grupo y de las 
organizaciones participantes en la red, lo cual se ha relacionado con la confianza interorganizacional y con liderazgo en los sectores de producción (Inkpen \& Dinur, 1998; Müller, 2012; Mursitama, 2006).

El ultimo tipo, el liderazgo de empoderamiento, tiene como comportamientos, promover el auto liderazgo y la autonomía, fomentar el trabajo en equipos, establecer desarrollo autónomo y de relaciones de trabajo y en las situaciones requeridas principalmente cuando se necesita fortalecer el conocimiento de los seguidores y participantes de una red así como cuando se necesita desarrollar nuevos líderes.

Este tipo de liderazgo es útil en os contextos de relaciones entre organizaciones en las acciones de conocimiento compartido principalmente en el mantenimiento y fortalecimiento de oportunidades de intercambio, cuando se ha posicionado una red y se quiere fortalecer la estrategia de liderar un sector y competir con conocimiento nuevo, con innovación y tecnología de última generación.

De acuerdo con Häcki \& Lighton (2001) y Nambisan \& Sawhney (2007), los orquestadores o líderes de la interrelación pueden desde esta posición de empoderamiento diseñar al interior de la red procedimientos tecnológicos que fortalezcan al grupo y a cada uno de los participantes, por ejemplo, mediante la instalación de plataformas electrónicas y el aprovechamiento de las tecnologías de comunicación e información.

Por último se considera que al ser la generación y el intercambio de conocimiento un fenómeno sobre el que definitivamente no existe una exclusiva forma de analizarse y sobre el que diversas variables intervienen haciendo de este un proceso dinámico del que emergen múltiples posibilidades, se propone un abordaje desde el liderazgo complejo el cual se inscribe dentro de los denominados sistemas adaptativos complejos (Lichtenstein, Uhl-Bien, Marion, Orton \& Schreiber, 2006).

Según esta perspectiva, el liderazgo puede ser entendido como un proceso dinámico que emerge de la interacción entre personas y transciende las capacidades individuales y grupales. Este modelo llevado al nivel de redes interorganizacionales posibilitaría el entendimiento del liderazgo como una propiedad emergente de las interacciones de las organizaciones que conforman la red; tales interacciones se realizan con base en reglas explicitas o implícitas de intercambio (Uhl-Bien, \& Marion, 2008) y para el caso de la generación de conocimiento en los contextos interorganizacionales se posibilitaría la comprensión dinámica y extensa de muchos determinante y factores involucrados desde la adquisición, la distribución, la interpretación, el almacenamiento de la información y la extensión de la misma, también favorecería la comprensión dinámica y multivariada de las diversas posibilidades de participación como la colaboración la competencia, el compromiso, la evitación y factores que también están involucrados y son cambiantes como la perdurabilidad de las redes, la confianza, los límites y los cambios en la capacidad de aprender.

\section{Conclusiones y perspectivas de investigación}

Se presentaron aquí elementos que posibilitan formular estudios futuros para conocer mejor las relaciones y dependencias entre lo que lo que se logra en las interrelaciones como rentas relacionales y lo que hacen los líderes en la interrelación, particularmente en el factor de conocimiento compartido.

El liderazgo en la relaciones interorganizacionales está determinado por diferentes variables (por ejemplo; el carácter jerárquico o heterárquico, el tipo de relación de cooperación establecida, el número de organizaciones participantes, el sector en el que se establecen las redes, entre otros) y puede asumir uno u otro estilo de acuerdo con la situación, el contexto y el momento de desarrollo que lleve la interrelación organizacional.

La perspectiva relacional sirve como modelo de análisis de los contextos interorganizacionales y como marco para identificar la función del liderazgo en los diferentes factores que componen la interrelación como son los activos de la relación, los recursoscapacidad complementarios, la gobernanza efectiva y las rutinas de conocimiento compartido. 
En el análisis y desarrollo de redes interorganizacionales particularmente cuando la dirección y la gestión del conocimiento compartido abordado, es importante tener en cuenta las características y el momento en el que se encuentra la relación así como las rutinas y las situaciones en la que el conocimiento surge.

El liderazgo de los procesos de compartir conocimiento requiere de en algunos casos posturas directivas y de conducción a objetivos particulares y específico pero en otras situaciones y circunstancias de asumir que la complejidad es una característica no solo delas relaciones sino del conocimiento en sí.

Es necesario proponer estudios que identifiquen las variables que determinan tanto la acción del liderazgo sobre el establecimiento de relaciones como de la incidencia de las relaciones en el liderazgo de las redes interorganizacionales.

Los contextos interorganizacionales pueden darse de diferentes maneras y analizarse desde distintas perspectivas y condiciones, los modelos exclusivos y modelos particulares de la relaciones entre liderazgo y conocimiento compartido pueden ser limitados.

Se sugiere involucrar el concepto de flexibilidad en el estudio de los tipos y características del liderazgo en los contextos interorganizacionales.

De otro lado, es pertinente e importante seguir desarrollando investigación empírica sobre liderazgo interorganizacional y comparar los hallazgos con los de la investigación clásica sobre liderazgo interpersonal con el fin de poner de relieve similitudes y diferencias.

Es necesario continuar con los esfuerzos por conocer las variables y relaciones que determinan la cultura de trabajo en red, la generación de aprendizaje y capacidades absorbentes que permee todas las esferas del quehacer empresarial y en definitiva determine nuevos patrones de comportamiento.

Es importante estudiar que las organizaciones también aprenden a través de contactos en la que no tienen relaciones estrechas o con las que no se es- tablecen relaciones de colaboración o cooperación formalmente.

Se sugiere estudiar cuando las redes interorganizacionales no funcionan y el conocimiento compartido no se produce o no genera rentas relacionales y seguir ampliando la comprensión de la gestión de estas relaciones y la exploración de su valor, sobre todo como herramienta de aprendizaje.

Los estudios futuros también podrían dirigirse a investigar cuales de los atributos individuales son más relevantes para el liderazgo de una red y que relaciones existen entre las características del liderazgo y la generación de confianza, compromiso, apropiación de conocimiento, cambio cultural en las redes de colaboración.

Se recomienda estudiar más allá de las rutinas de conocimiento compartido el aprendizaje como conjunto de capacidades, habilidades, conocimiento e información que la firma receptora adquiere, acumula o incrementa como resultado de las interacciones propias de la relación con otra firma fuente del conocimiento y como resultado de las acciones de liderazgo.

\section{Referencias}

Arbaiza, L. (2009). Liderazgo y complejidad: una nueva visión para la gestión. América Economía. Recuperado de http:// mba.americaeconomia.com/articulos

Barney, J. (1991). Firm resources and sustained competitive advantage. Journal of Management, 17, 99-120.

Barringer, B. \& Harrison, J. (2000). Walking a tightrope: Creating value through interorganizational relationships. Journal of Management, 26, 367-403.

Bass, B. \& Riggio, R. (2006). Transformational Leadership. Mahwah, NJ: Lawrence Erlbaum Associates.

Castro, A. (2006). Teorías implícitas del liderazgo, contexto y capacidad de conducción. Anales de Psicología, 22, 89-97.

Contreras, F. (2008). Liderazgo: perspectivas de investigación y desarrollo. International Journal of Psychological Research 1(2), 64-72.

Cox, J., Pearce, C. \& Sims, H. (2011). Toward a broader leadership development agenda: Extending the traditional transactional transformational duality by developing directive, empowering, and shared leadership skills. En: S.E. Murphy \& R.E. Riggio (Eds.), The future of leadership development: 161-179. New York: Taylor \& Francis Group.

Dhanaraj, C. \& Parkhe, A. (2006). Orchestrating innovation networks. Academy of Management Review, 31, 659-669. 
Dinh, J., Lord, R., Gardner, W., Meuser, J., Liden, R. \& Hu, J. (2014). Leadership theory and research in the new millennium: Current theoretical trends and changing perspectives. The Leadership Quarterly, 25(1), 36-62.

Dini, M. (2010). Competitividad, redes de empresas y cooperación empresarial. CEPAL Serie Gestión Pública, 72

Doshi, V. \& Khokle, P. (2011). Impact of Inter-organizational Relationships on Organizational Learning. Research and Publications. Indian Institute of Management. Recuperado de http://www.iimahd.ernet.in/assets/snippets

Doz, Y. \& Hamel, G. 1998. Alliance advantage. Boston: Harvard Business School Press.

Dyer, J. \& Nobeoka, K. (2000). Creating and managing a highperformance knowledge-sharing network: The Toyota Case. Strategic Management Journal, 21, 345-367.

Dyer, J. \& Singh, H. (1998). The relational view: Cooperative strategy and sources of interorganizational competitive advantage. Academy of Management Review, 23, 660-679.

Galaskiewicz, S. \& Shatin, D. (1981). Leadership and networking among neighbourhood human service organizations. Administrative Science Quarterly, 26, 434-48.

García, V., Jiménez, M. \& Gutiérrez, L. (2012). Transformational leadership influence on organizational performance through organizational learning and innovation. Journal of Business Research, 65(7), 1040-1050.

Grueso, M., Gómez, J. \& Garay, L. (2010). Redes empresariales e innovación: el caso de una red del sector cosmético en Bogotá (Colombia). Estudios Gerenciales, 27(118), 189-203.

Häcki, R. \& Lighton, J. (2001). The future of the networked company. The McKinsey Quarterly, 3, 26-39.

Huxham, C. \& Vangen, S. (2000). Leadership in the shaping and implementation of collaboration agendas:how things happen in a (not quite) joined-up world. Academy of Management Journal, 43, 1159-1175.

Inkpen, A. \& Dinur, A. (1998). Knowledge management processes and international joint ventures. Organization Science, 9, 454-468.

Knight, L. (2002). Network learning: Exploring learning by interorganizational networks. Human Relations, 55(4), 427-454.

Kraatz, M. (1998). Learning by association? Interorganizational Networks and Adaptation to Environmental Change. Academy of Management Journal, 41(6), 621-643.

Lavie, D. (2006). The competitive advantage of interconnected firms: An extension of the resource-based view. Academy of Management Review, 31, 638-58.

Levinson, N. \& Asahi, M. (1996). Cross-national alliances and interorganizational learning. Organizational Dynamics, 24, 51-63.

Lichtenstein, B., Uhl-Bien, M., Marion, R., Orton, J. \& Schreiber, C. (2006). Complexity leadership theory: An interactive perspective on leading in complex adaptive systems. Paper 8 (4), 2-12.

Lupano, M. \& Castro, A. (2005). Estudios sobre el liderazgo. Teorías y evaluación. Psicodebate. Psicología, Cultura y Sociedad. 6, 107-121.
Matinheikki, J., Artto, K., Peltokorpi, A. \& Rajala, R. (2016) Managing inter-organizational networks for value creation in the front-end of projects. International Journal of Project Management, 34(7), 1226-1241.

Mendoza, I., Ortíz, M. \& Parker, H. (2007). Dos décadas de investigación y desarrollo en liderazgo transformacional. Revista del Centro de Investigación. Universidad La Salle, 7, 1665-8612.

Mesquita, L., Anand, J. \& Brush, T. (2008). Comparing the resource-based and relational views. Strategic Management Journal, 29, 913-41.

Müller, G. (2012). Leadership in interorganizational networks: A literature review and suggestions for future research. International Journal of Management Reviews, 14, 428-443.

Mursitama T. (2006). Creating relational rents: The effect of business groups on affiliated firms' performance in Indonesia. Asia Pacific Journal of Management, 23, 537-557.

Nambisan, S. \& Sawhney, M. (2007). Buyer's guide to the innovation bazaar. Harvard Business Review, 109-118.

Paglis, L. \& Green, S. (2002). Leadership self-efficacy and managers' motivation for leading change. Journal of Organizational Behavior, 23, 215-235.

Peroune, L. (2007). Tacit knowledge in the workplace: the facilitating role of peer relationships. Journal of European Industrial Training, 31(4), 244-258.

Porter, M. (1980). Competitive strategy. New York. Free Press.

Powell, W., Koput, K. \& Smith-Doerr, L. (1996). Interorganizational collaboration and the locus of innovation: Networks of learning in biotechnology. Administrative Science Quarterly, $41,116-145$

Rumelt, R. (1991). How much does industry matter? Strategic Management Journal, 12, 167-185.

Sánchez, J. \& Jiménez, P. (2007). La cooperación empresarial como estrategia de crecimiento: motivos de su formación, ventajas $e$ inconvenientes. En: Decisiones basadas en el conocimiento y en el papel social de la empresa: Memorias XX Congreso anual de AEDEM (p. 30). Asociación Española de Dirección y Economía de la Empresa (AEDEM).

Scott, T. \& Thomas, C. (2015). Do collaborative groups enhance interorganizational networks? Public Performance \& Management Review, 38(4), 654-683.

Uhl-Bien, M. \& Marion, R. (2008). Complexity leadership - A framework for leadership in the twenty-first century. En M. Uhl-Bien \& M. Russ (Eds.), Complexity leadership: 6-24. Charlotte, NC: Information Age Publishing.

Verdu, F. \& Reinert, M. (2016). Interorganizational relationships networks in internationalization process of exporting companies. Revista de Negócios, 20(3), 7-15.

Vincent, R. \& Byrne, A. (2006). Enhancing learning in development partnerships. Development in Practice, 16 (5), 385-399.

Yukl, G. (2006). Leadership in Organizations. Upper Saddle River, NJ: Prentice Hall. 